\title{
Chronische myeloische Leukämie - Pathophysiologie und Diagnostik
}

\author{
O. J. Stoetzer ${ }^{1}$ \\ M. Hentrich ${ }^{2}$ \\ C. Salat ${ }^{1}$
}

\section{Chronic myeloid leukemia - pathophysiology and diagnosis}

\section{Glossar}

\section{$\mathrm{ABL}=$ Abelson}

Ara-C $=$ Cytarabin

$\mathrm{BCR}=$ reakpoint-Cluster-Region

$\mathrm{CML}=$ Chronische myeloische Leukämie

$\mathrm{HU}=$ Hydroxyurea

IFN- $\alpha=$ Interferon- $\alpha$

$\mathrm{KM}=$ Knochenmark

$\mathrm{KO}=$ Körperoberfläche

$\mathrm{PB} \quad=$ peripheres Blut

Ph-Chromosom $=$ Philadelphia-Chromosom

SZT = Stammzelltransplantation

Die chronische myeloische Leukämie (CML) ist eine klonale myeloproliferative Erkrankung, die durch die maligne Entartung und unkontrollierte Expansion hämatopoetischer Stammzellen entsteht. Die Inzidenz der CML liegt bei ca. zwei Fällen pro 100000 Einwohner. Damit macht diese Erkrankung etwa 1520\% aller Leukämien im Erwachsenenalter aus. Eine CML kann in jedem Lebensalter auftreten, der Altersgipfel liegt jedoch im fünften und sechsten Dezennium (medianes Erkrankungsalter: 48 Jahre). Männer erkranken häufiger als Frauen (M:W=3:2) $(3,5,8)$.

Die Ätiologie der CML ist weitgehend unklar. Erbliche oder infektiöse Ursachen sind nicht bekannt. Eine Häufung tritt nach radioaktiven Bestrahlungen auf. Chemische Leukämogene werden diskutiert.

\section{Pathogenese}

Die Pathogenese der CML ist relativ gut aufgeklärt. Diese Kenntnisse spielen eine wichtige Rolle für das Verständnis der Diagnostik und neuerdings auch der Therapie der Erkrankung. Bei 9095\% der Patienten mit klinisch typischer CML lässt sich das Philadelphia-Chromosom $(\mathrm{Ph})$ nachweisen. Das $\mathrm{Ph}-\mathrm{Chromosom}$ ist ein verkürztes Chromosom 22, das durch einen reziproken Austausch von genetischem Material zwischen den langen Armen von Chromosom 9 und 22 entsteht. Dieser Vorgang wird als Translokation $\mathrm{t}(9 ; 22)$ bezeichnet. Der Bruchpunkt auf Chromosom 9 liegt im Bereich des Abelson Proto-Onkogens (c-ABL), welches normalerweise für das 145 kD ABL-Protein - eine Tyrosinkinase (p145 ${ }^{\mathrm{ABL}}$ ) - kodiert. Das verkürzte ABL-Gen wird auf Chromosom 22 in die Region des Breakpoint Cluster Region (BCR) Gens eingefügt, es entsteht ein BCR-ABL-Fusionsgen. Dieses kann bei bis zu 98\% der Patienten mit CML nachgewiesen werden. Je nachdem, welche Exons fusionieren, entstehen unterschiedliche chimäre Gene. Bei der CML entsteht am häufigsten das p210 ${ }^{\mathrm{BCR}-}$ ${ }^{A B L}$ Genprodukt, welches gegenüber dem normalen p145 ${ }^{\mathrm{ABL}}$ eine deutlich erhöhte Tyrosinkinaseaktivität besitzt. Vermittelt über Regulatorproteine führt $\mathrm{p} 210^{\mathrm{BCR}-\mathrm{ABL}}$ zu einer gesteigerten Mitoserate und damit zur unkontrollierten Proliferation der CML-Zellen. Das Ph-Chromosom und die BCR-ABL-Translokation lassen sich in allen proliferierenden Zellen der Hämatopoese, sowie in BLymphozyten nachweisen. Dagegen gehört nur ein Teil der TLymphozyten zum malignen Klon (1,2,7,9).

${ }^{1}$ Hämato-Onkologische Schwerpunktpraxis am Rotkreuzplatz, München

${ }^{2}$ Krankenhaus München-Harlaching; IV. Medizinische Abteilung 
Die Symptomatik der CML ist meist uncharakteristisch. In 50\% der Fälle wird die Erkrankung als Zufallsbefund über eine Blutbildbestimmung oder eine Oberbauchsonographie diagnostiziert. Bei Patienten mit symptomatischer Erkrankung stehen uncharakteristische Befunde wie Abgeschlagenheit, Schwäche, Inappetenz, Gewichtsverlust, Knochenschmerzen und Oberbauchbeschwerden im Vordergrund. Bei etwa 70\% der Patienten findet sich eine palpabel vergrößerte Milz (2). Extramedulläre Infiltrate und Fieber sind ungünstige Zeichen und finden sich nur selten.

\section{Diagnostik}

Neben der Anamnese, der körperlichen Untersuchung (Hepatosplenomegalie, extramedulläre Infiltrate, Blutungszeichen) und der Abdomen-Sonographie sind für die Diagnose der CML vor allem Laboruntersuchungen erforderlich:

1. Komplettes Blutbild/Differentialblutbild: Leitbefund der CML ist eine Leukozytose, die sich bei 98\% der Patienten nachweisen lässt. Leukozytenzahlen bis über $600 \times 10^{9} / 1$ sind keine Seltenheit. Im Differentialblutbild findet sich eine kontinuierliche Linksverschiebung mit dem Auftreten von unreifen Vorstufen der Myelopoese (Metamyelozyten, Myelozyten, Promyelozyten, Myeloblasten). Häufig liegt eine Eosinophilie und Basophilie vor. In über 30\% der Fälle besteht eine Thrombozytose. Der Index der alkalischen Leukozytenphosphatase (ALP) ist meistens erniedrigt.

2. Knochenmarkaspiration- und Biopsie: Es findet sich eine ausgeprägte Hyperplasie der Myelopoese und häufig auch der Megakaryopoese. Gelegentlich zeigt sich bereits bei der Erstdiagnose ein erhöhter Fasergehalt. Dieser Anteil nimmt im Verlauf der Erkrankung zu. Häufig lassen sich glykolipidspeichernde Makrophagen, die als Pseudo-Gaucher-Zellen bezeichnet werden, darstellen.

3. Chromosomenanalyse: Das Ph-Chromosom ist bei $90-95 \%$ der Patienten mit klinisch typischer CML nachweisbar. In etwa $10 \%$ der Fälle werden weitere Aberrationen gefunden, wie ein zweites Ph-Chromosom, das Isochromosom 17 oder eine Trisomie 8 oder 19.

4. Nachweis des BCR/ABL-Transkripts: Der molekularbiologische Nachweis des BCR-ABL-Transkripts erfolgt in der Regel über eine Multiplex-RT-PCR. Dadurch kann die BCR/ ABL-Translokation in 3-5\% der Ph-negativen Fälle nachgewiesen werden. Die quantitative PCR eignet sich für die Verlaufsbeurteilung und den Nachweis einer minimalen Resterkrankung (minimal residual disease, MDR), insbesondere nach allogener Zelltransplantation (2). Mit Hilfe der FISH-Technik (Fluoreszenz-in-situ-Hybridisierung) kann die Translokation auch morphologisch in einzelnen Zellkernen markiert werden.

5. Laborchemische Bestimmung von LDH und Harnsäure: Hervorgerufen durch den hohen Zellumsatz findet sich häufig eine Hyperurikämie, sowie eine Erhöhung der LDH.

6. Immunzytologie: Liegt eine Blastenkrise vor, so kann mit Hilfe der Immunzytologie zwischen lymphatischem und myeloischem Phänotyp unterschieden werden.
Tab. 1 Kriterien der chronischen Phase, Akzeleration und Blastenkrise (WHO-Klassifikation 2001).

\begin{tabular}{|c|c|c|}
\hline & Kriterien & Mediane Dauer \\
\hline Chronische Phase & Blasten $\leq 10 \%$ im KM oder PB & 4-6 Jahre \\
\hline Akzeleration & $\begin{array}{l}\text { - Blasten } \geq 10 \% \text { im PB oder KM } \\
\text { - Basophilie/Eosinophilie } \geq 20 \% \\
\text { - Thrombozytose }>1000 \times 10^{9} / / \text {, } \\
\text { therapierefraktär } \\
\text { - Thrombopenie }<100 \times 10^{9} / / \text {, } \\
\text { nicht Therapie-assoziiert } \\
\text { - Neue chromosomale Aberrationen } \\
\text { - Progrediente Leukozytose, } \\
\text { therapierefraktär } \\
\text { - Progrediente Splenomegalie, } \\
\text { therapierefraktär }\end{array}$ & Monate \\
\hline Blastenschub & $\begin{array}{l}\text { - Blasten } \geq 20 \% \text { im PB oder KM } \\
\text { - Zytologisch oder histologisch } \\
\text { gesicherte extra-medulläre } \\
\text { Infiltrate } \\
\text { - Große Blasten-Cluster in der } \\
\text { KM-Biopsie }\end{array}$ & 2-9 Monate \\
\hline
\end{tabular}

\section{Diagnosesicherung}

Ein typischer Blutbild- und Knochenmarkbefund in Verbindung mit dem Nachweis des Ph-Chromosoms bzw. des BCR-ABL-Fusionstranskripts sichern die Diagnose der CML.

\section{Differenzialdiagmose}

Bei Ph-negativen Fällen ohne BCR-ABL-Nachweis ist die differentialdiagnostische Abgrenzung anderer myeloproliferativer Erkrankungen (Polycythaemia vera [PV], idiopathische Myelofibrose [IMF], essentielle Thrombozythämie [ET], sowie der chronischen myelomonozytären Leukämie schwierig. Hier können der ALP-Index, der bei den anderen myeloproliferativen Erkrankungen normal oder erhöht ist, die Knochenmarkhistologie sowie letztlich der Krankheitsverlauf richtungsweisend sein.

\section{Stadieneinteillung}

Die CML ist charakterisiert durch einen zwei- oder dreiphasigen Krankheitsverlauf. Die Mehrheit der CML-Fälle (85\%) wird in der chronischen Phase diagnostiziert (2). Während dieser Phase ist der Krankheitsverlauf relativ stabil und therapeutisch gut kontrollierbar. Nach 4-6 Jahren geht die Erkrankungen entweder direkt oder über eine so genannte akzelerierte Phase in die Blastenphase (Blastenschub, Blastenkrise) über (Tab.1). Der Zeitpunkt der Akzeleration ist bei Diagnosestellung im Einzelfall nicht vorhersagbar. Ca. 30\% der Blastenkrisen sind phänotypisch B-lymphoblastisch. T-zelluläre Marker werden sehr selten gefunden (6). Die Prognose des Blastenschubs ist nach wie vor sehr ungünstig. 
kurzgefasst: Die chronische myeloische Leukämie wird

häufig als Zufallsbefund bei einer Blutbildkontrolle diagnostiziert. Für die Diagnose richtungweisend ist das Differenzialblutbild, der morphologische Knochenmarkbefund, sowie der Nachweis des Ph-Chromosoms bzw. der BCR/ABL-Translokation. In 85\% der Fälle liegt bei Diagnosestellung eine chronische Phase vor.

\section{Prognosescore}

Basierend auf Daten von Interferon-Therapie-Studien wurde ein neuer Prognosescore entwickelt, der auf den Faktoren Alter, Milzgröße, Eosinophilie, Basophilie, Myeloblastenanteil und der Thrombozytenzahl basiert. Eine Berechnung des Scores über verschiedene Multiplikatoren kann via Internet erfolgen (www.pharmacoepi.de). Es werden drei Risikogruppen unterschieden (Niedrigrisiko $\leq 780$, intermediäres Risiko 781-1479, Hochrisiko $\geq 1480$ ). Das 6-Jahres-Gesamtüberleben liegt hierbei in der Niedrigrisiko-Gruppe bei ca. 76\%, in der Gruppe mit intermediärem Risiko bei ca. $48 \%$ und in der Hochrisiko-Gruppe nur bei ca. $25 \%$ (4).

Wichtigste verlaufsabhängige Prognoseparameter sind das Erreichen einer kompletten hämatologischen Remission mit der Normalisierung des Blutbildes, der Milzgröße und der CML-bedingten Symptome sowie das Erreichen einer zytogenetischen Remission.

\section{Fazit für die Praxis}

Die chronische myeloische Leukämie kann in jedem Lebensalter auftreten, die Symptomatik ist oft uncharakteristisch. Zur Diagnosesicherung muss neben dem Differentialbludbild eine Knochenmarkpunktion einschließlich zytogenetischer (Ph-Chromosom) und molekularbiologischer (BCR/ABL) Untersuchung durchgeführt werden.

Autorenerklärung: Die Autoren erklären, dass sie keine finanziellen Verbindungen mit einer Firma haben, deren Produkt in dem Artikel eine wichtige Rolle spielt (oder mit einer Firma, die ein Konkurrenzprodukt vertreibt).

\section{Literatur}

1 Bartram CR, de Klein A, Hagemeijer A et al. Translocation of c-abl oncogene correlates with the presence of a Philadelphia chromosome in chronic myelocytic leukaemia. Nature 1983; 306: 277-280

2 Faderl S, Talpaz M, Estrov Z, Kantarjian HM. Chronic Myelogenous Leukemia: Biology and Treatment. Ann Intern Med 1999; 131: $207-$ 219

3 Faderl S, Talpaz M, Estrow Z, O`Brien S, Kurzrock R, Kantarjian HM. The biology of chronic myeloid leukemia. N Engl J Med 1999; 341: 164-172

4 Hasford J, Pfirrmann J, Hehlmann R et al. A new prognostic score for survival of patients with chronic myeloid leukemia treated with interferon alfa. J Nat Cancer Inst 1998; 90: 850-858

5 Hehlmann R, Heimpel H, Hasford J and the German CML Study Group. Randomized comparison of interferon-alpha with busulfan and hydroxyurea in chronic myelogenous leukaemia (CML). Blood 1994; 84: 4064-4077

6 Kantarijan HM, Keating MJ, Talpaz M et al. Chronic myelogenous leukemia in blast crisis. Analysis of 242 patients. Amer J Med 1987; 83: 445-454

7 Rowley JD. A new consistent chromosomal abnormality in chronic myelogenous leukaemia identified by quinacrine fluorescence and Giemsa staining. Nature 1973; 243: 290-293

8 Sawyers CL. Chronic myeloid leukemia. N Engl J Med 1999; 340: 1330-1339

9 Witte ON et al. Role of the BCR/ABL oncogene in human leukemia. Cancer Res 1993; 53: 485-489 